\title{
Escala de Braden: instrumento norteador para a prevenção de úlceras por pressão
}

\author{
Braden Scale: guiding instrument for pressure ulcer prevention
}

Escala de Braden: instrumento guía para la prevención de úlceras de presión

Jeane Silvestri Wechi1, Lúcia Nazareth Amante1, Nádia Chiodelli Salum¹, Eliane Matos', Tatiana Martins

\begin{abstract}
RESUMO
O estudo objetivou avaliar o risco para o desenvolvimento de úlcera por pressão em pacientes internados em clínica médica. Trata-se de um estudo observacional, quantitativo, realizado entre 14 de maio e 14 de agosto de 2012 por meio da aplicação da escala preditiva de Braden. Foram avaliados 120 pacientes internados em um hospital federal de uma capital do Sul do Brasil. Utilizou-se para a coleta de dados um instrumento para avaliação de risco: Escala de Braden. Os resultados apontaram que a média de idade dos pacientes foi de 52 anos. Foram realizadas 435 avaliações. O estudo demonstrou que os idosos e os pacientes dependentes apresentaram, respectivamente, alto e altíssimo risco para o desenvolvimento de úlcera por pressão, demandando, portanto, cuidados especiais durante a internação. Confirmou-se a efetividade da escala de Braden para avaliação de risco de desenvolvimento de úlceras por pressão, uma vez que possibilita a identificação precoce dos riscos e a adoção de medidas preventivas, já que, durante sua utilização, não houve formação de úlceras por pressão.
\end{abstract}

DESCRITORES: Estomaterapia. Cuidados de enfermagem. Úlcera por pressão. Medição de risco.

\begin{abstract}
The study aimed at evaluating the risk for developing pressure sores in clinical medical inpatients. This is an observational, quantitative study, conducted between May 14 and August 14, 2012, through the application of predictive scale of Braden. We evaluated 120 patients admitted to a federal hospital in a southern capital of Brazil. It was used for data collection an instrument for risk assessment: Braden Scale. The results showed that the mean age of patients was 52 years. Four hundred and thirty-five evaluations were performed. The study showed that elderly and dependent patients had, respectively, high and very high risk for developing pressure sores, demanding, therefore, special care during hospitalization. It confirmed the effectiveness of the Braden Scale for assessing the risk of developing pressure sores, as it enables the early identification of risks and the adoption of preventive measures, since, while using it, there was no formation of pressure ulcers.
\end{abstract}

DESCRIPTORS: Stomatherapy. Nursing care. Pressure ulcer. Risk Assessment.

\section{RESUMEN}

El estudio tuvo como objetivo evaluar el riesgo del desarrollo de úlceras por presión en pacientes hospitalizados en clínica médica. Se trata de un estudio observacional y cuantitativo, realizado entre 14 de mayo y 14 de agosto de 2012, a través de la aplicación de la escala predictiva de Braden. Se evaluaron 120 pacientes ingresados en un hospital federal de una capital en el Sur de Brasil. La recolección de datos utilizó un instrumento para la evaluación del riesgo: la Escala de Braden. Los resultados muestran que la edad media de los pacientes fue de 52 años. Se realizaron 435 evaluaciones. El estudio demostró que los pacientes mayores y dependientes tenían, respectivamente, alto y muy alto riesgo de desarrollar úlceras por presión, exigiendo, por lo tanto, atención especial durante la hospitalización. Se confirmó la eficacia de la escala de Braden para evaluar el riesgo del desarrollo de úlceras por presión, ya que permite la identificación temprana de los riesgos y la adopción de las medidas preventivas, porque, durante su uso, no se formaron úlceras por presión.

DESCRIPTORES: Estomaterapia. Atención de enfermería. Úlcera por presión. Evaluacion de riesgos.

Universidade Federal de Santa Catarina - Florianópolis (SC), Brasil.

Endereço para correspondência: Rua Altamiro Guimarães, 330, apto. 1.302 - Centro - CEP: 88015-510 - Florianópolis (SC), Brasil -

E-mail: jeanewechi@hotmail.com

Artigo recebido em: 17/11/2015 - Aceito para publicação em: 23/07/2016. 


\section{INTRODUÇÃO}

A úlcera por pressão é um dano localizado na pele e/ou nos tecidos moles subjacentes, geralmente sobre uma proeminência óssea ou relacionada ao uso de dispositivo médico ou outro artefato. A úlcera pode se apresentar em pele íntegra ou como úlcera aberta, e pode ser dolorosa. A úlcera ocorre como resultado da pressão intensa e/ou prolongada em combinação com o cisalhamento. A tolerância do tecido mole à pressão e ao cisalhamento também pode ser afetada pelo microclima, pela nutrição, pela perfusão, por comorbidades e por sua condiçãa ${ }^{1}$.

Está relacionada a um ou vários fatores de risco, tais como: imobilidade; integridade musculoesquelética alterada; talas, órteses e próteses; aparelhos restritivos; estado alterado da consciência; sensibilidade reduzida; umidade excessiva; emagrecimento; esquema inadequado de reposicionamento; superfície de apoio inadequada; sobrecarga em áreas corporais de risco; higiene corporal inadequada e falta de hidratação/ proteção da pele ressecada ${ }^{2,3}$.

O sistema de classificação atualizado inclui as seguintes definições:

- Úlcera por pressão Estágio 1: pele íntegra com eritema que não embranquece.

- Úlcera por pressão Estágio 2: perda da pele em sua espessura parcial com exposição da derme.

- Úlcera por pressão Estágio 3: perda da pele em sua espessura total.

- Úlcera por pressão Estágio 4: perda da pele em sua espessura total e perda tissular.

- Úlcera por pressão Não Classificável: perda da pele em sua espessura total e perda tissular não visível.

- Úlcera por pressão Tissular Profunda: descoloração vermelho-escura, marrom ou púrpura, persistente e que não embranquece ${ }^{1}$.

\section{A National Pressure Ulcer Advisory Panel (NPUAP)} também define Úlcera por Pressão Relacionada a Dispositivo Médico e Úlcera por Pressão em Membranas Mucosas.

A incidência de úlcera por pressão em ambiente hospitalar tem sido objeto de estudos da Enfermagem, conforme pode ser constatado em pesquisa realizada em um hospital de médio porte no Estado de Minas Gerais que objetivou identificar a incidência e a prevalência de úlcera por pressão. No estudo, foram admitidos 1.096 pacientes, dos quais 477 (43,5\%) foram avaliados pela Escala de Braden (EB). Oitenta pacientes apresentaram úlcera por pressão, dos quais 43 $(53,7 \%)$ a desenvolveram durante o período de internação; a prevalência geral de úlcera foi de $7,29 \%$ e a incidência, de 3,9\%. Conclui-se, portanto, que a maior incidência de úlcera por pressão foi registrada na Unidade de Terapia Intensiva (UTI), ressaltando-se que, como profissional da saúde, o enfermeiro tem destaque na equipe multiprofissional para o tratamento de úlceras ${ }^{4}$.

A prevenção e o tratamento das úlceras por pressão ainda constituem um desafio para a Enfermagem. No entanto, estudos realizados em UTIs demonstram a relevância da aplicação de instrumentos preditivos na prevenção dessa complicação e na garantia do cuidado com a qualidade. Estudo realizado em um hospital público do Estado de Pernambuco, utilizando a EB, evidenciou que a maioria dos pacientes internados na UTI apresentou risco elevado ou moderado para desenvolver úlcera por pressão $0^{5}$.

Com o objetivo de auxiliar os profissionais de saúde a identificarem e avaliarem os riscos de o paciente desenvolver úlcera por pressão durante a internação, foram desenvolvidas e utilizadas diferentes propostas de escalas para avaliar o risco de úlcera tissular. Algumas delas são reconhecidas mundialmente, como as escalas de Norton, Gosnell, Waterlow e Braden ${ }^{6}$.

Cada qual com suas especificidades, as escalas funcionam como instrumentos de avaliação de risco ou de medidas que possibilitam a prevenção de lesões de pele.

Neste estudo, optou-se pela utilização da EB por se entender que ela atende aos objetivos propostos e ao considerar estudos que mostram que sua utilização apresenta boa sensibilidade para a avaliação clínica de risco para o desenvolvimento de úlcera por pressão, quando comparada com outra escala dessa natureza ${ }^{7}$.

A EB apresenta seis variáveis para avaliação em seis subescalas: Percepção Sensorial, Umidade, Atividade, Mobilidade, Nutrição, Fricção e força de Cisalhamento ${ }^{8}$. Cada subescala é pontuada de 1 a 4 , exceto a variável Friçção e Cisalhamento, que pontua de 1 a 3 . O escore total pode variar de 6 a 23 pontos, sendo os pacientes classificados da seguinte forma: risco muito alto (escores iguais ou menores a 9 pontos), risco alto (escores de 10 a 12 pontos), risco moderado (escores de 13 a 14 pontos), baixo risco (escores de 15 a 18 pontos) e sem risco (escores de 19 a 23 pontos).

A EB pode subsidiar o trabalho do enfermeiro, a quem cabe identificar os pacientes com risco de desenvolver úlcera por pressão $0^{10}$. 
Outro aspecto fundamental na prevenção de úlcera por pressão é a capacitação dos profissionais de enfermagem. Um estudo, que analisou o conhecimento sobre a prevenção de úlcera por pressão da equipe de enfermagem que presta assistência direta ao paciente adulto e idoso em um hospital universitário de Minas Gerais, constatou que tanto o enfermeiro quanto o técnico de enfermagem apresentaram desempenho menor que o esperado ${ }^{11}$. Outro estudo, realizado em um hospital público da região mineira, constatou que, embora a equipe de enfermagem conhecesse as diferentes práticas recomendadas para a prevenção e o tratamento da úlcera por pressão, o enfermeiro desconhecia seu papel no cuidado ao paciente portador de feridas ${ }^{12}$. Os achados na literatura apontam, portanto, para a necessidade de atualização permanente da equipe de enfermagem na prevenção e nos cuidados com a úlcera por pressão.

A condição apontada na literatura é percebida também na instituição em que este estudo foi realizado, especificamente na unidade de internação de clínica médica de um hospital universitário. Nesse local, não existe registro sobre a incidência, a prevalência e as características clínico-demográficas dos pacientes em risco ou com úlcera por pressão. Também não há padronização relacionada à avaliação da pele do paciente, embora se perceba a preocupação dos profissionais com a prevenção dessa complicação. Nesse sentido, acredita-se que a utilização de uma escala preditiva para úlcera por pressão, como a EB, permitirá identificar o paciente de risco e conhecer seu perfil clínico-demográfico, de modo a estabelecer medidas preditivas que garantam sua segurança e a assistência de enfermagem.

\section{OBJETIVO}

Identificar de acordo com a Escala de Braden, o potencial de risco para o desenvolvimento de úlcera por pressão em pacientes internados na unidade de clínica médica.

\section{MÉTODO}

Estudo observacional, quantitativo, realizado em uma unidade de clínica médica de um hospital geral universitário situado no Sul do Brasil, entre 14 de maio e 14 de agosto de 2012. Foram incluídos no estudo 120 pacientes internados nesse período, os quais aceitaram participar, considerando-se os critérios a seguir: idade maior de 18 anos, lúcidos e orientados ou acompanhados de familiares, com ou sem úlcera por pressão no momento da avaliação. Foram excluídos os pacientes que estavam sem acompanhantes. Para a avaliação do potencial de risco, utilizou-se a $\mathrm{EB}^{10}$.

Foram coletados também os dados de procedência, especialidade médica que estava atendendo os pacientes, diagnóstico, data de admissão e alta.

Os enfermeiros foram capacitados para a utilização da EB na avaliação diária do risco de úlcera por pressão por meio de quatro encontros presenciais, organizados de acordo com o Arco de Maguerez ${ }^{13}$, que abordaram seu conhecimento prévio de úlcera por pressão, das escalas preditivas, com ênfase na $E B$, bem como do planejamento do cuidado para as medidas preventivas. Nesse momento, foi definida a dinâmica de aplicação segundo o grau de dependência. Para os técnicos e auxiliares de enfermagem, foram realizados quatro encontros presenciais que trataram dos cuidados e das medidas preventivas para úlceras por pressão.

Concluída a etapa de capacitação e iniciada a coleta de dados, todos os 120 pacientes internados no período da coleta foram avaliados. Estes foram avaliados no momento da internação e reavaliados, segundo critérios de dependência, conforme a dinâmica preestabelecida com os enfermeiros: os pacientes dependentes diariamente, os semidependentes a cada dois dias e os independentes a cada sete dias. Assim, foram realizadas 435 avaliações.

De acordo com os critérios acordados com os enfermeiros, o paciente foi considerado independente quando realizava sem auxílio atividades da vida diária, como andar, comer e fazer a higiene pessoal; semidependente quando necessitava de auxilio para realizar as mesmas atividades; e dependente quando não apresentava condições de realizar as atividades da vida diária.

Os dados coletados foram processados e organizados em uma planilha eletrônica no Microsoft Office Excel ${ }^{\circledR}$ 2012, sendo posteriormente analisados estatisticamente à luz da literatura pertinente. Para o cálculo da incidência de úlcera por pressão, foi considerada a razão entre o número de casos novos no período da coleta de dados multiplicado por cem e o número de pessoas expostas ao risco no mesmo período . $^{8}$

Para manter o anonimato, os pacientes foram identificados de acordo com a presença ou não de úlcera por pressão, permanecendo as seguintes siglas: $\mathrm{NN}$ para os pacientes que foram admitidos e receberam alta sem úlcera por pressão; $\mathrm{SN}$ para os pacientes que foram admitidos com úlcera por pressão e receberam alta sem úlcera por pressão; SS para os pacientes que foram admitidos e receberam alta com úlcera por pressão. 
O estudo foi aprovado pelo Comitê de ética de Pesquisa com Seres Humanos, com Certificado de Apresentação para Apreciação Ética (CAAE) no 01320912.8.0000.0121.

\section{RESULTADOS}

Em relação ao perfil sociodemográfico, os 120 participantes do estudo tinham média de idade de 52 anos, sendo 80 $(66,66 \%)$ do sexo feminino e $40(33,34 \%)$ do sexo masculino. Quanto à especialidade médica responsável pela internação, o maior percentual foi registrado para Clínica Médica (26,89\%), seguido de Pneumologia (18,49\%), Reumatologia (11,76\%), Gastroenterologia (11,76\%), Neurologia (9,24\%), Endocrinologia (8,40\%), Cardiologia (7,56\%) e Nefrologia (5,88\%). Na Figura 1, apresenta-se a relação entre o risco de desenvolvimento de úlcera por pressão e a dependência dos pacientes segundo o número de avaliações realizadas.

Observa-se que, nos 3 meses, 435 avaliações foram realizadas entre os pacientes independentes, semidependentes e dependentes. Os pacientes independentes se apresentaram sem risco para úlcera por pressão em 171 avaliações (40\%) e com baixo risco em 3 avaliações (1\%). Os pacientes semidependentes foram avaliados sem risco em 73 (17\%) dos casos avaliados, com baixo risco em 42 (9\%) dos casos, risco moderado em 7 e risco alto em 6 (2\%) avaliações.
Os pacientes dependentes foram avaliados com baixo risco em 27 (7\%), risco moderado em 21 (5\%), risco alto em 60 (14\%) e risco muito alto em 25 (5\%) dos casos.

O tempo de internação médio foi calculado em 18 dias, e 95 pacientes permaneceram internados por até 20 dias. Destaca-se a permanência de 18 pacientes por um período de 21 a 40 dias e de 6 pacientes por um período de 41 a 60 dias de internação.

Observa-se a predominância de pacientes com idade maior que 70 anos, seguidos por indivíduos com idade entre 51 e 60 anos, internados na referida unidade. Com relação ao risco para o desenvolvimento de úlcera por pressão, encontraram-se 271 pacientes (63\%) com baixo risco; 67 (15\%) com risco muito alto; 66 (15\%) com risco alto e 31 (7\%) com risco moderado.

Os dados referentes à distribuição e ocorrência de úlcera por pressão durante o período de internação são apresentados na Figura 2.

Observou-se que, do total de pacientes internados, 112 (93\%) não desenvolveram úlcera por pressão (NN) durante o período de internação, 7 (6\%) já foram internados com úlcera por pressão $(\mathrm{SN})$ e permaneceram com ela até a alta hospitalar e 1 (1\%) foi internado com úlcera por pressão (SS), a qual, no momento da alta hospitalar, apresentava-se reepitelizada. Destaca-se que nenhum paciente desenvolveu úlcera por pressão durante a hospitalização.

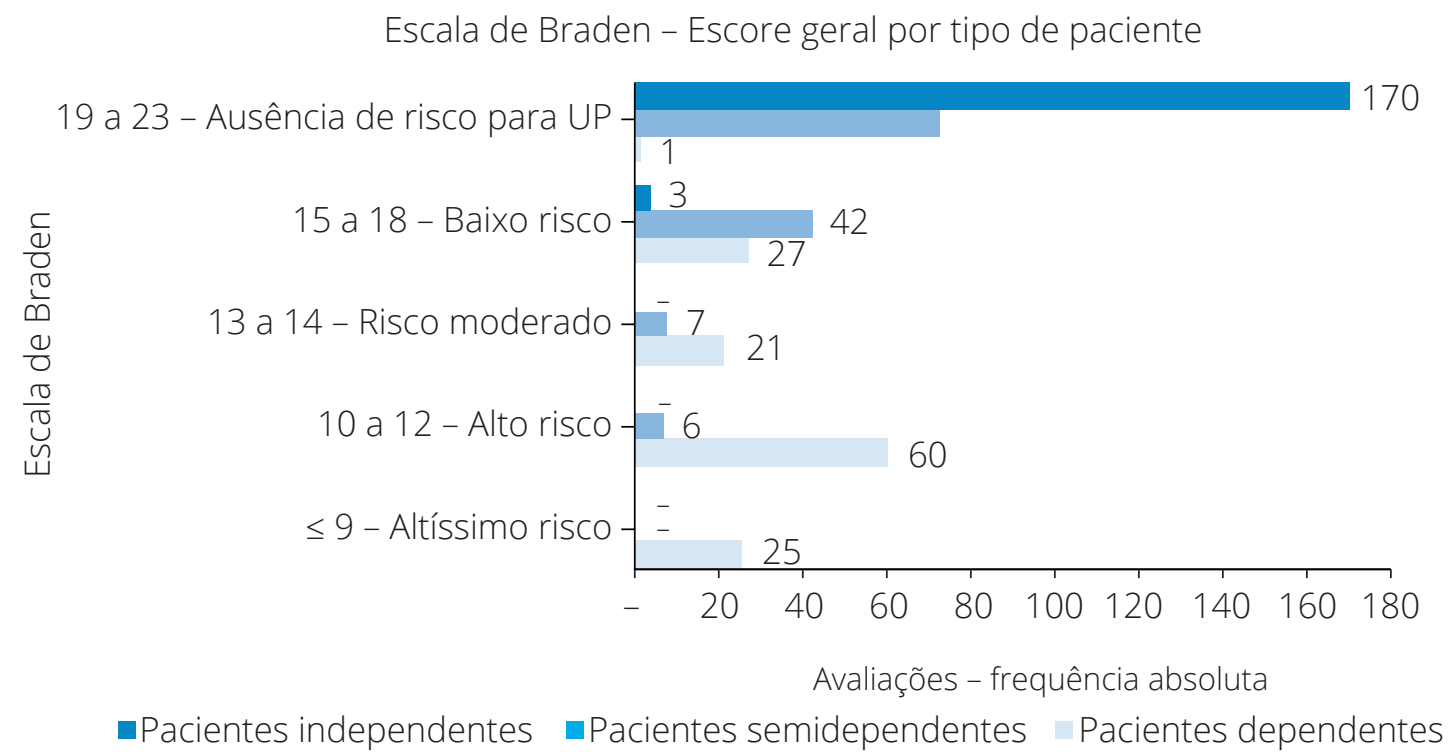

Fonte: banco de dados dos autores.

Figura 1. Distribuição da quantidade de avaliações de acordo com o grau de dependência dos cuidados de enfermagem, Florianópolis, SC, 2012. (Abaixo do primeiro parágrafo dos Resultados). 


\section{DISCUSSÃO}

A análise da avaliação do risco de desenvolvimento de úlcera por pressão nos 120 pacientes que participaram do estudo demonstrou que a faixa etária acima de 70 anos apresenta risco muito alto para a complicação.

Esse dado corrobora a literatura, que esclarece que o envelhecimento é um processo gradual no qual o indivíduo sofre alterações morfológicas, fisiológicas, bioquímicas e psicológicas, com perda progressiva da capacidade de se adaptar e se manter em equilíbrio com o meio, ampliando sua vulnerabilidade e sua predisposição a desenvolver processos patológicos. Cabe considerar que as manifestações ligadas aos fatores cronológico e biológico trazem consequências em todas as dimensões da vida e da saúde do idoso ${ }^{14}$.

No que diz respeito às alterações na pele, acontece o comprometimento do fluxo sanguíneo pela diminuição da vascularização, que leva à redução da oxigenação, hidratação e nutrição, reduzindo, por sua vez, os níveis de proteína e albumina, que, associados às doenças crônicas, aumentam a susceptibilidade para o desenvolvimento de úlcera por pressão $0^{2,3,15}$.

Nessa perspectiva, uma pesquisa realizada com 140 pacientes internados em 22 UTIs de hospitais públicos e privados de Belo Horizonte concluiu que 64\% deles, com 60 anos ou mais, apresentavam risco para o desenvolvimento de úlcera por pressão ${ }^{16}$. Outro estudo, realizado com 74 pacientes em
UTIs de um hospital universitário de nível terciário na cidade de São Paulo, encontrou resultado semelhante, quando se associou a ocorrência de úlcera por pressão à média de idade mais elevada, de 60 anos $^{17}$. Nesse sentido, infere-se que a idade, o estado de saúde e a mobilidade são fatores que contribuem para a presença de lesões de pele.

A especialidade que mais motivou a internação foi a de clínica médica, seguida pela pneumologia, o que está em conformidade com a literatura, que afirma serem os setores de clínica médica e UTIs os locais onde se encontraram pacientes portadores de úlcera por pressão. Isso se deve ao tempo prolongado de internação e à associação da gravidade, complexidade e grau de dependência dos pacientes com doenças infecciosas (pneumonia e infecção do trato urinário, redução da percepção sensorial causada por medicamentos sedativos, analgésicos e relaxantes musculares, acarretando menor reação à pressão excessiva) ${ }^{17,18}$.

Cabe salientar que a avaliação dos pacientes foi realizada diariamente e, com isso, houve modificação de seu estado e de sua propensão ao desenvolvimento de úlcera por pressão, uma vez que a $\mathrm{EB}$ foi aplicada em consonância com a classificação de dependência dos pacientes para o cuidado de enfermagem nas 435 avaliações efetuadas. A avaliação contínua do paciente influenciou a ocorrência de classificação de um número maior de pacientes em semidependentes e independentes, uma vez que o quadro se modificava a cada avaliação, da internação à alta hospitalar.

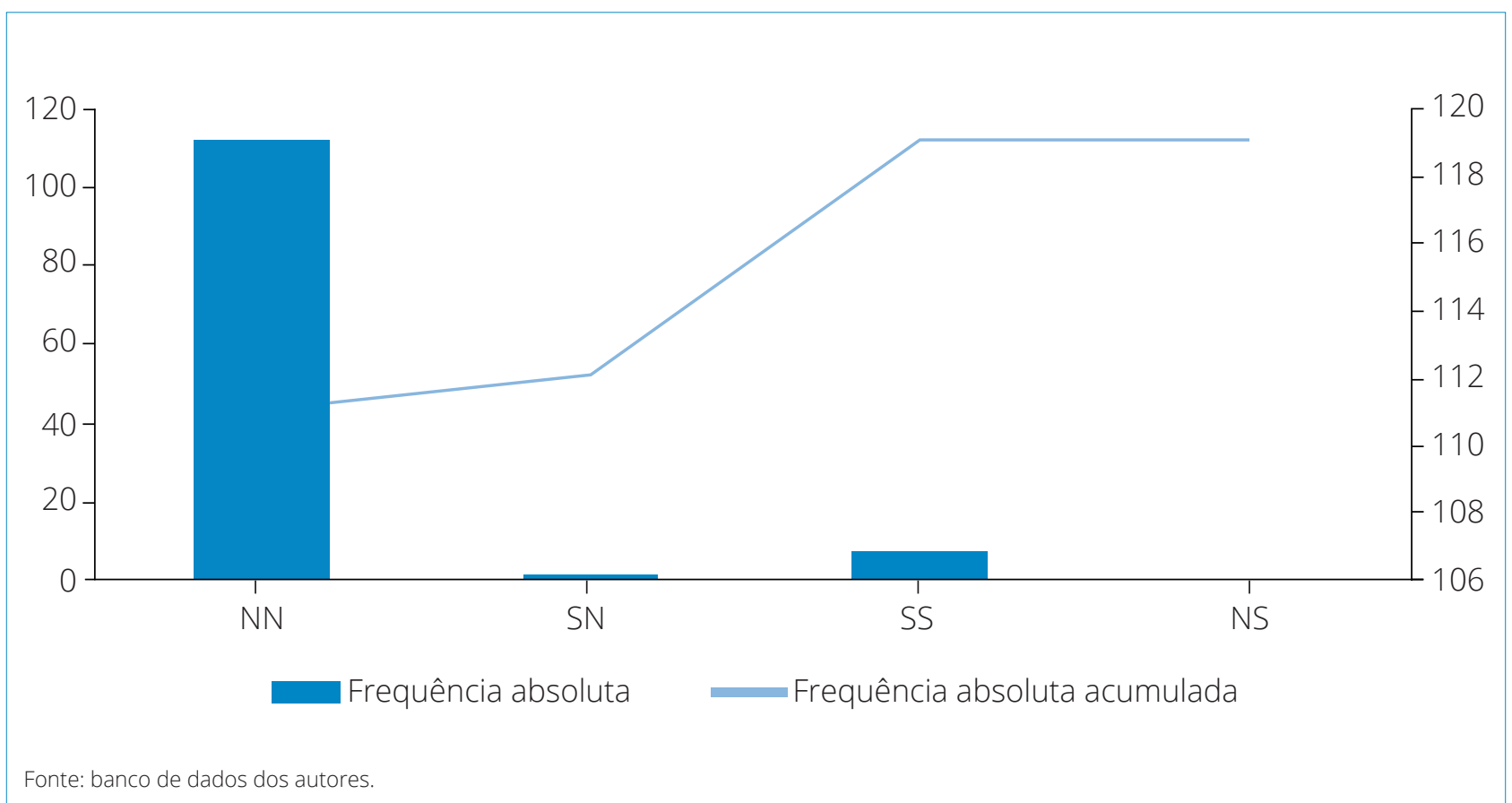

Figura 2. Distribuição dos pacientes de acordo com a presença de úlcera por pressão na internação, durante a hospitalização e na alta, Florianópolis, SC, 2012. 
A classificação dos pacientes em relação à dependência dos cuidados de enfermagem se mostrou efetiva e em consonância com os resultados de um estudo realizado em um hospital universitário de Porto Alegre, que analisou a associação entre uma classificação de pacientes e a EB em unidades de internação, com 2.562 pacientes $^{19}$. Também foi comprovada a relação entre a dependência do paciente e a ocorrência de úlcera por pressão em pacientes adultos internados. Os autores confirmaram que a utilização de instrumentos de avaliação facilita a identificação do risco e o estabelecimento precoce de medidas de prevenção.

A média de dias de internação, assim como o estado dos pacientes, pode ter influência sobre a não ocorrência de úlcera por pressão. Um estudo realizado com 690 pacientes em um hospital público de Joinville (SC) identificou a média e o tempo médio de desenvolvimento das úlceras por pressão, que ocorrem geralmente a partir de 13 dias de internação para um paciente cirúrgico, 8 dias para intercorrências clínicas e 10 dias para pacientes de UTIs. O tempo médio de internação aumenta o risco para o desenvolvimento de úlcera por pressão ${ }^{20}$.

Embora neste estudo as avaliações tenham identificado um número maior de pacientes com baixo risco para o desenvolvimento de úlcera por pressão, ao serem somadas as avaliações de risco moderado, risco alto e risco muito alto, constata-se a necessidade de implementar cuidados de enfermagem para a prevenção de úlcera por pressão em 164 casos.

Como não houve desenvolvimento de úlcera por pressão no período da coleta de dados, sendo a incidência igual a zero, infere-se a eficácia dos cuidados de prevenção implementados, quais sejam,o uso de colchão piramidal; a massagem de conforto; a elevação dos calcanhares com o uso de travesseiro debaixo da panturrilha; e a mudança de decúbito ${ }^{3,10}$. Embora o colchão piramidal aumente o conforto, mas não reduza a pressão, ele foi utilizado por ser um dos meios disponíveis na instituição, associado à massagem de conforto e à mudança de decúbito.

A inexistência de novos casos de úlcera por pressão na instituição estudada, com a adoção de medidas de prevenção para essa complicação, confirma que as recomendações de prevenção devem ser aplicadas a todos os indivíduos que apresentem risco de desenvolvimento de úlcera por pressão, em todos os grupos etários. Para a avaliação do risco de desenvolvimento de úlcera por pressão, o enfermeiro pode utilizar diariamente, além das escalas preditivas, a avaliação clínica e a anamnese, o que facilita o processo de sistematização da assistência por meio da avaliação, da elaboração do diagnóstico de enfermagem, da prescrição e da evolução de enfermagem. O momento da realização do banhotem se constituído um excelente momento para examinar a pele e avaliar a eficácia de medidas preventivas adotadas, tais como controle do excesso de pressão sobre as eminências ósseas, uso de soluções para manter a integridade da pele, redistribuidores de pressão, entre outros ${ }^{21}$. As intervenções prescritas devem ser adotadas pelos profissionais de saúde envolvidos no cuidado de pacientes e pessoas vulneráveis que se encontrem em ambiente hospitalar, em cuidados continuados e em instituições de longa permanência, independentemente de seu diagnóstico e das necessidades de cuidados ${ }^{22}$.

Em relação às vantagens da utilização da EB para a avaliação, a prevenção e o controle das úlceras por pressão, diversos estudos identificam aspectos positivos nesse sentido ${ }^{12,13}$. A EB é considerada um instrumento de fácil utilização e eficiente para predizer o risco de úlcera por pressão, com sensibilidade e especificidade adequadas ${ }^{9}$.

A utilização da EB para avaliar o risco de úlcera por pressão se demonstrou positiva durante a realização deste estudo, pois todos os pacientes foram avaliados sistematicamente, o que favoreceu a prevenção e a recuperação. Nesse sentido, foram desenvolvidas intervenções de enfermagem para cada subescala da escala de Braden, tais como: percepção sensorial e umidade, mobilidade e atividade, nutrição, friç̧ão e cisalhamento, que auxiliaram o enfermeiro a planejar a assistência ao paciente.

Entretanto, embora se perceba a conscientização e a preocupação dos enfermeiros com relação ao cuidado e à aplicação de escalas preditivas em pacientes que apresentam úlcera por pressão, ainda é necessário organizar capacitações constantes para a prevenção da complicação em instituições de saúde.

\section{CONCLUSÃO}

O estudo demonstrou que os idosos e os pacientes dependentes apresentaram, respectivamente, alto e altíssimo risco para o desenvolvimento de úlcera por pressão, demandando, portanto, cuidados especiais durante a internação.

Os dados que emergiram do presente estudo reafirmam a EB como instrumento preditivo para a avaliação do risco de úlcera por pressão, pois ela possibilita a realização do exame físico, especialmente a inspeção da integridade da pele. Ressalta-se positivamente que, durante o período de aplicação da EB, a incidência de úlcera por pressão foi igual a zero e uma úlcera por pressão previamente existente cicatrizou-se a partir da avaliação e da implementação de cuidados específicos. Sendo assim, a 
aplicação diária da EB contribuiu para a sistematização da assistência de enfermagem e, embora não tenha sido objeto do estudo, para a atualização da equipe de enfermagem com vistas à programação e à implementação de cuidados que mantenham a integridade da pele dos pacientes.
Entende-se que novos estudos que contemplem um número maior de pacientes e a inclusão de outras instituições de saúde devam ser incentivados com o objetivo de ampliar a aplicabilidade da EB como ferramenta preditiva de cuidados com a integridade da pele.

\section{REFERÊNCIAS}

1. National Pressure Ulcer Advisory Panel (NPUAP). Pressure Injury Stages [Internet]; 2016. Staging Consensus Conference that was held April 8-9, 2016. Disponível em: http://www. npuap.org/resources/educational-and-clinical-resources/ npuap-pressure-injury-stages/. Acesso em: 30 abr. 2016.

2. Irion GL. Feridas - novas abordagens, manejo clínico e atlas em cores. 2. ed. Rio de Janeiro: Guanabara Koogan; 2012.

3. Pott FS, Ribas JD, Silva OBM, Souza TS, Danski MTR, Marineli MJ. Algoritmo de prevenção e tratamento de úlcera por pressão. Cogitare Enferm [Internet]. 2013;18(2):238-44. Disponível em: <http://dx.doi.org/10.5380/ce.v18i2.26085>. Acesso em: 05 dez. 2013.

4. Melo L, Gonçalves O, Vieira DS. Incidência e prevalência de úlcera por pressão dos usuários atendidos em um hospital de médio porte. Perquirere. 2015;12(1):137-49. Disponível em: http://perquirere.unipam.edu.br. Acesso em: 06 nov. 2015.

5. Silva EWNL, Araújo RA, Oliveira EC, Falcão VTFL. Aplicabilidade do protocolo de prevenção de úlcera de pressão em unidade de terapia intensiva. Rev Bras Ter Intensiva. 2010; 22(2):175-85. Disponível em: http://www.scielo.br/pdf/rbti/ v22n2/a12v22n2.pdf. Acesso em: 21 mai. 2015.

6. Pinto EN, Souza SROS, Oliveira ES, Figueiredo NMA, Gomes LLO. Sinal de alerta para úlceras por compressão e a enfermagem. In: Silva RCL, Figueiredo NMA, Meireles IB (Orgs.). Feridas: fundamentos e atualizações em enfermagem. 3. ed. São Caetano do Sul: Yendis; 2011. p. 413-54.

7. Borghardt AT, Prado TN, Araújo TM, Rogenski NMB, Bringuente MEO. Avaliação das escalas de risco para úlcera por pressão em pacientes críticos: uma coorte prospectiva. Rev Latino-Am Enfermagem. 2015;23(1):28-35. Disponível em: $\quad$ http://www.scielo.br/pdf/rlae/v23n1/pt_0104-1169rlae-23-01-00028.pdf>. Acesso em: 04 out. 2015.

8. Hans M, Bitencourt JVOV, Pinheiro F. Additional risk factors related to Braden Scale: a risk for pressure ulcers. Enferm Foco [Internet]. 2011;2(4):222-25 Disponível em: <http:// revista.portalcofen.gov.br/index.php/enfermagem/article/ view/188/124>. Acesso em: 15 mai. 2013.

9. Prevention Plus. Home of the Braden Scale [Internet]; 2010. Disponivel: <http://www.bradenscale.com>. Acesso em: 05 dez. 2014.

10. Caliri MHL. Úlcera por pressão/recomendações para a prevenção. Disponível em: <http://www2.eerp.usp.br/site/ grupos/feridascronicas/index.php?option=com_content\&vi ew=article\&id=16\&ltemid=24>. Acesso em: 01 out. 2015.

11. Miyazaki MY, Caliri MHL, Santos CB. Conhecimento dos profissionais de enfermagem sobre prevenção da úlcera por pressão. Rev Latino-Am Enfermagem. 2010;18(6):[10 telas]. Disponível em: <http://www.scielo.br/pdf/rlae/v18n6/ pt_22.pdf>. Acesso em: 01 out. 2015.

12. Martins DA, Soares FFR. conhecimento sobre prevenção e tratamento de úlceras de pressão entre trabalhadores de enfermagem em um hospital de Minas gerais. Cogitare Enferm. 2008;13(1):83-7. Disponível em: <http://ojs.c3sl. ufpr.br/ojs/index.php/cogitare/article/viewArticle/11956>. Acesso em: 04 out. 2015.

13. Bordenave JD, Pereira AM. Estratégias de ensinoaprendizagem. 30. ed. Petrópolis: Vozes; 2009.

14. Lisboa CR. Risco para úlcera por pressão em idosos institucionalizados [Dissertação]. Belo Horizonte: Universidade Federal de Minas Gerais, Escola de Enfermagem, Programa de Pós-Graduação em Enfermagem. Disponível em: <http://www.enf.ufmg.br/pos/defesas/648M. PDF>. Acesso em: 01 out. 2015.

15. Borges EL, Fernandes FP. Úlcera por pressão. IN: Domansky RC, Borges EL (Orgs.). Manual para prevenção de lesões de pele: recomendações baseadas em evidências. Rio de Janeiro: Rubio; 2012. p. 151-218.

16. Gomes FSL, Bastos MAR, Matozinhos FP, Temponi HR, Meléndez GV. Avaliação de risco para úlcera por pressão em pacientes críticos. Rev Esc Enferm USP [Internet]. 2011;45(2):313-18. Disponível em: <http://www.scielo.br/ pdf/reeusp/v45n2/v45n2a01.pdf>. Acesso em: 20 out. 2012.

17. Cremasco MF, Wenzel F, Sardinha FM, Zanei SSV, Whitaker IY. Úlcera por pressão: risco e gravidade do paciente e carga de trabalho de enfermagem. Acta Paul Enferm. 2009;22(Especial - 70 Anos):897. Disponível em: <http://www.scielo.br/pdf/ape/ v22nspe/en_11.pdf>. Acesso em: 04 out. 2015.

18. Matos LS, Duarte NLV, Minetto RC. Incidência e prevalência de úlcera por pressão no CTI de um Hospital Público do DF. Rev Eletr Enf [Internet]. 2010;12(4):719-26. Disponível em: <http:// dx.doi.org/10.5216/ree.v12i4.8481>. Acesso em: 17ago. 2016.

19. Urbanetto JS, Santos MACN, Poltozi AF, Pechansky ALC, Hax G, Custódio A. A relação entre a dependência de cuidados, riscos e úlcera por pressão. Enferm Foco [Internet]. 2012;3(4):198-201. Disponível em: <http:// revista.portalcofen.gov.br/index.php/enfermagem/article/ view/384/175>. Acesso em: 21 nov. 2011.

20. Moro A, Maurici A, Valle JB, Zaclikevis VR, Kleinubing Junior $H$. Avaliação dos pacientes portadores de lesão por pressão internados em hospital geral. Rev Assoc Med Bras. 2007;53(4):300-4. Disponível em: <http:// www.scielo.br/scielo.php?script=sci_arttext\&pid =S0104-42302007000400013>. Acesso em: 20 fev. 2016.

21. Ferreira JDL, Aguiar ESS, Lima CLJ, Brito KKG, Costa MML, Soares MJGO. Ações preventivas para úlcera por pressão em idosos com declínio funcional de mobilidade física no âmbito domiciliar. Estima. 2016;14(1):36-42.

22. Brasil. Ministério da Saúde. Anvisa. Fiocruz. Protocolo para prevenção de úlcera por pressão. Disponível em: <http://www.anvisa.gov.br/hotsite/segurancadopaciente/ documentos/julho/PROTOCOLO\%20ULCERA\%20POR\%20 PRESS\%C3\%830.pdf>. Acesso em 09 jul. 2013. 